\title{
Dose dependence of ferromagnetism in Co-implanted ZnO
}

\author{
Numan Akdogan, ${ }^{1, a)}$ Hartmut Zabel, ${ }^{1}$ Alexei Nefedov, ${ }^{1, b)}$ Kurt Westerholt, ${ }^{1}$ \\ Hans-Werner Becker, ${ }^{2}$ Safak Gök, ${ }^{3}$ Rustam Khaibullin, ${ }^{4}$ and Lenar Tagirov ${ }^{4}$ \\ ${ }_{1}^{1}$ Institut für Experimentalphysik/Festkörperphysik, Ruhr-Universität Bochum, D-44780 Bochum, Germany \\ ${ }^{2}$ Institut für Physik mit Ionenstrahlen, Ruhr-Universität Bochum, 44780 Bochum, Germany \\ ${ }^{3}$ Lehrstuhl für Angewandte Festkörperphysik, Ruhr-Universität Bochum, 44780 Bochum, Germany \\ ${ }^{4}$ Kazan Physical-Technical Institute of RAS, 420029 Kazan, Russia and Kazan State University, 420008 \\ Kazan, Russia
}

(Received 19 November 2008; accepted 12 January 2009; published online 26 February 2009)

We have studied the structural, magnetic, and electronic properties of Co-implanted $\mathrm{ZnO}(0001)$ films grown on $\mathrm{Al}_{2} \mathrm{O}_{3}(11 \overline{2} 0)$ substrates for different implantation doses and over a wide temperature range. Strong room temperature ferromagnetism is observed with magnetic parameters depending on the cobalt implantation dose. A detailed analysis of the structural and magnetic properties indicates that there are two magnetic phases in Co-implanted $\mathrm{ZnO}$ films. One is a ferromagnetic phase due to the formation of long range ferromagnetic ordering between implanted magnetic cobalt ions in the $\mathrm{ZnO}$ layer and the second one is a superparamagnetic phase, which occurs due to the formation of metallic cobalt clusters in the $\mathrm{Al}_{2} \mathrm{O}_{3}$ substrate. Using $\mathrm{x}$-ray resonant magnetic scattering, the element specific magnetization of cobalt, oxygen, and $\mathrm{Zn}$ was investigated. Magnetic dichroism was observed at the $\mathrm{Co} L_{2,3}$ edges as well as at the $\mathrm{O} K$ edge. In addition, the anomalous Hall effect is also observed, supporting the intrinsic nature of ferromagnetism in Co-implanted ZnO films. (C) 2009 American Institute of Physics. [DOI: 10.1063/1.3082080]

\section{INTRODUCTION}

$\mathrm{ZnO}$ is a II-VI semiconductor with a wide band gap of about $3.4 \mathrm{eV}$. The stable crystal structure of $\mathrm{ZnO}$ is the wurtzite structure (hexagonal, with $a=3.25 \AA$ and $c$ $=5.12 \AA),{ }^{1}$ in which each atom of zinc is surrounded by four oxygen atoms in tetrahedral coordination. The magnetic transition metal (TM) doped $\mathrm{ZnO}$ is interesting from the viewpoint of forming a transparent ferromagnetic material, and it has the potential to be a highly multifunctional material with coexisting ferromagnetic, semiconducting, and magnetooptical properties. Theoretical predictions of room temperature ferromagnetism in TM-doped $\mathrm{ZnO}$ (Refs. 2-4) have initiated a number of experimental works on these systems as a potential oxide-based diluted magnetic semiconductor material. The first observation of ferromagnetism in Co-doped $\mathrm{ZnO}$ was reported by Ueda et al. ${ }^{5}$ They prepared $\mathrm{Zn}_{1-x} \mathrm{Co}_{x} \mathrm{O}$ thin films on sapphire substrates using pulsed laser deposition (PLD) technique with $x$ varying between 0.05 and 0.25 . Following these initial theoretical and experimental reports, different growth methods have been used to deposit $\mathrm{Co:ZnO}$ films, including radio-frequency (rf) magnetron co-sputtering, ${ }^{6}$ PLD using a KrF laser, ${ }^{7-14}$ combinatorial laser molecular beam epitaxy, ${ }^{15,16}$ and sol-gel method, ${ }^{17,18}$ as well as ion implantation. ${ }^{19}$ Sapphire has been widely used as substrate due to the small mismatch (2\%) between (0001) oriented $\mathrm{ZnO}$ and $\mathrm{Al}_{2} \mathrm{O}_{3}(11 \overline{2} 0)$ substrates. In addition to co-

\footnotetext{
a) Author to whom correspondence should be addressed. Present address: Department of Physics, Gebze Institute of Technology, Gebze, 41400 Kocaeli, Turkey. Electronic addresses: numan.akdogan@ ruhr-uni-bochum.de. ${ }^{b)}$ Present address: Lehrstuhl für Physikalische Chemie I, Ruhr-Universität Bochum, 44780 Bochum, Germany.
}

balt, other $3 d$ transition elements have also been used for doping, including $\mathrm{Mn},{ }^{5,15,20-22} \mathrm{Ni},{ }^{5,11,15} \mathrm{~V},{ }^{11,15,23} \mathrm{Cr},{ }^{5,11}$ and also Fe. ${ }^{5,11,15,24}$

Various solubility limits for $\mathrm{Co}$ in $\mathrm{ZnO}$ were reported by different groups. Prellier et al..$^{10}$ determined a solubility limit of about 10 at. \% in PLD-grown films. Park et al. ${ }^{25}$ reported that cobalt nanoclusters start to form for $x \geq 12$ at. \% in samples grown by sol-gel and rf sputtering techniques. Lee et al. ${ }^{17}$ observed some undefined Bragg peaks for a cobalt concentrations higher than 25 at. \%. Kim et al. ${ }^{8}$ showed that the solubility limit is less than 40 at. \% in PLD-grown films. Ueda et al. ${ }^{5}$ claimed that the solubility limit is lower than 50 at. \% and they clearly observed a phase separation into $\mathrm{ZnO}-$ and $\mathrm{CoO}$-rich phases in the film prepared using $\mathrm{Zn}_{0.5} \mathrm{Co}_{0.5} \mathrm{O}$ targets. These controversial results from different research groups are likely due to different growth techniques used and/or due to different growth conditions such as oxygen pressure and deposition temperature. Recently, we have reported that using ion implantation cobalt concentrations of up to 50 at. $\%$ in $\mathrm{ZnO}$ are possible without cobalt cluster formation. ${ }^{19}$ This high concentration is attributed to the properties of ion implantation, which allows doping of transition metals beyond their equilibrium solubility limits. ${ }^{26}$ Regarding the magnetic properties of Co-doped $\mathrm{ZnO}$ films, while several groups including ourself have observed room temperature ferromagnetism for 50 at. $\%,{ }^{19}$ $25-30$ at. $\%,{ }^{14,17}$ and lower ${ }^{10,11,13,27}$ Co concentrations, others reported the absence of ferromagnetism at room temperature. $8,15,25$

In this paper we report detailed studies using various experimental techniques for the investigation of the structural, magnetic, and electronic properties of Co-implanted $\mathrm{ZnO}$ films grown on sapphire substrates and for different 


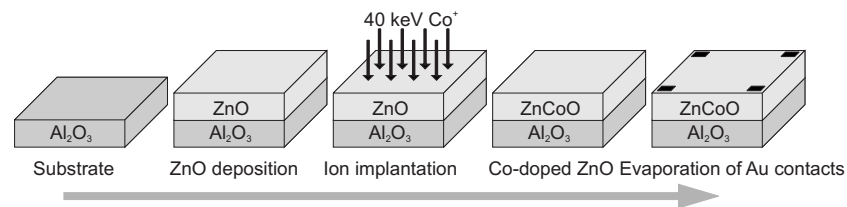

FIG. 1. Sample preparation stages for Co-implanted $\mathrm{ZnO} / \mathrm{Al}_{2} \mathrm{O}_{3}$ films.

cobalt concentrations. Rutherford backscattering spectroscopy (RBS) and x-ray diffraction (XRD) were used to determine the depth profile of implanted cobalt ions and to detect the formation of possible secondary phases such as metallic cobalt clusters. The magnetic properties of the films were characterized by the magneto-optical Kerr effect (MOKE), a superconducting quantum interference device (SQUID) magnetometer, as well as x-ray resonant magnetic scattering (XRMS) techniques. In order to determine the type and concentration of carriers in Co-implanted $\mathrm{ZnO}$ films, Hall effect measurements were also performed.

\section{SAMPLE PREPARATION}

About $35 \mathrm{~nm}$ thick $\mathrm{ZnO}(0001)$ films were grown on 10 $\times 10 \mathrm{~mm}^{2}$ epipolished single-crystalline $a$-plane $\mathrm{Al}_{2} \mathrm{O}_{3}(11 \overline{2} 0)$ substrates by rf $(13.56 \mathrm{MHz})$ sputtering of a $\mathrm{ZnO}$ target. ${ }^{28}$ The sputtering was carried out in an atmosphere of $5 \times 10^{-3}$ mbar pure $\operatorname{Ar}(99.999 \%)$ with a substrate temperature of $500{ }^{\circ} \mathrm{C}$. In order to increase the quality of $\mathrm{ZnO}$ films, the samples were annealed in an oxygen atmosphere with a partial pressure of up to 2000 mbars and a temperature of $800{ }^{\circ} \mathrm{C}$. After annealing, the $\mathrm{ZnO}$ samples were implanted with $40 \mathrm{keV} \mathrm{Co}{ }^{+}$ions with an ion current density of $8 \mu \mathrm{A} \mathrm{cm}^{-2}$ using the ILU-3 ion accelerator (Kazan Physical-Technical Institute of Russian Academy of Science). The sample holder was cooled by flowing water during the implantation to prevent the samples from overheating. The implantation dose varied in the range of $0.25-2.00 \times 10^{17}$ ions $\mathrm{cm}^{-2}$. After implantation, the samples were cut into square pieces and gold contacts were evaporated on the corners of the samples for Hall effect studies (Fig. 1). A list of the Co-implanted $\mathrm{ZnO}$ films used for the present study is given in Table I.

TABLE I. List of the $\mathrm{ZnO}$ films implanted with $40 \mathrm{keV} \mathrm{Co}^{+}$for varying $\mathrm{Co}$ ion dose.

\begin{tabular}{cc}
\hline \hline Sample & Dose $\left(\times 10^{17}\right.$ ion $\left.\mathrm{cm}^{-2}\right)$ \\
\hline 1 & 0.25 \\
2 & 0.50 \\
3 & 0.75 \\
4 & 1.00 \\
5 & 1.25 \\
6 & 1.50 \\
7 & 2.00 \\
\hline \hline
\end{tabular}

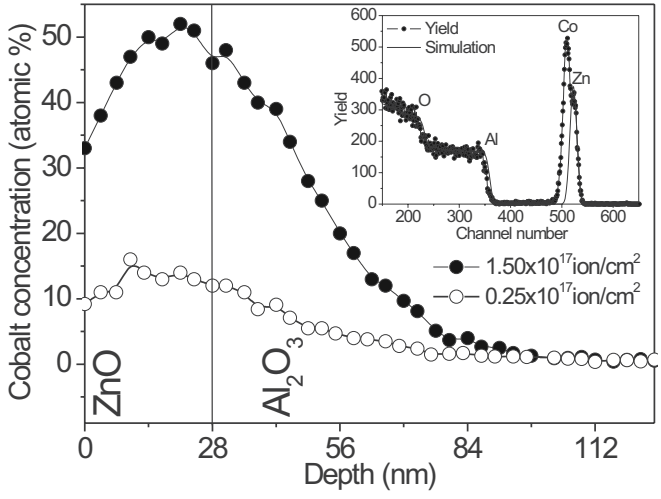

FIG. 2. Depth dependence of the cobalt concentration in $\mathrm{ZnO} / \mathrm{Al}_{2} \mathrm{O}_{3}$ implanted with Co ions with doses of $0.25 \times 10^{17}$ ions $\mathrm{cm}^{-2}$ (open symbols) and $1.50 \times 10^{17}$ ions $\mathrm{cm}^{-2}$ (black symbols), respectively. The inset shows the experimentally observed (symbols) and simulated (solid line) RBS spectra for sample 6 .

\section{EXPERIMENTAL RESULTS}

\section{A. Structural properties}

The depth dependence of the cobalt concentration in Coimplanted $\mathrm{ZnO} / \mathrm{Al}_{2} \mathrm{O}_{3}$ films was investigated using the $\mathrm{RBS}$ technique at the Dynamic Tandem Laboratory at the RuhrUniversität Bochum. The RBS data show both a maximum of cobalt concentration located close to the $\mathrm{ZnO} / \mathrm{Al}_{2} \mathrm{O}_{3}$ interface and an extended inward tail due to cobalt diffusion into the volume of the $\mathrm{Al}_{2} \mathrm{O}_{3}$ substrate (Fig. 2). We also noticed that after ion implantation the thickness of the $\mathrm{ZnO}$ layer has shrunk (e.g., from originally 35 to $28 \mathrm{~nm}$ for sample 6) due to ion sputtering of the substrate surface. According to the SRIM algorithm, ${ }^{29}$ the average implanted depth of $40 \mathrm{keV}$ Co ions in $\mathrm{ZnO} / \mathrm{Al}_{2} \mathrm{O}_{3}$ is about $20.4 \mathrm{~nm}$ with a straggling of $9.6 \mathrm{~nm}$ in the Gaussian-type depth distribution. However, because of the surface sputtering, ion mixing, and heating of the implanted region by the ion beam, a redistribution of the implanted cobalt ions compared to the calculated profile has to be taken into account.

High-angle XRD experiments provide information on the structural coherence of the films and in our case also of possible additional phases in the sample after ion implantation. Figure 3 shows high angle Bragg scans of the $\mathrm{ZnO}$ films before and after cobalt implantation. The data were taken using synchrotron radiation at the "Hamburg Synchrotron Radiation Laboratory" (HASYLAB) (for pure ZnO film) and at the "Dortmund Electron Accelerator" (DELTA) (for cobalt implanted $\mathrm{ZnO}$ films) with the energies of $8048 \mathrm{eV}$ and $11000 \mathrm{eV}$, respectively. Before implantation the XRD pattern consists of a very strong $\mathrm{Al}_{2} \mathrm{O}_{3}(11 \overline{2} 0)$ peak and a $\mathrm{ZnO}(0001)$ reflection to the left side. The $\mathrm{ZnO}$ peak is surround by thin film Laue oscillations, which are indicative for the high quality of the $\mathrm{ZnO}$ film. After implantation, the XRD diffraction pattern shows a (1010) reflection of the Co hcp structure on the right side of the sapphire substrate peak. The ion bombardment also causes an intensity reduction in the $\mathrm{ZnO}(0001)$ peak proportional to the implantation dose, indicating an increasing amount of lattice defects. Furthermore, after implantation we observe a shift of the $\mathrm{ZnO}$ (0001) peak to higher angles. Ueda et al. ${ }^{5}$ and Prellier et al. ${ }^{10}$ 


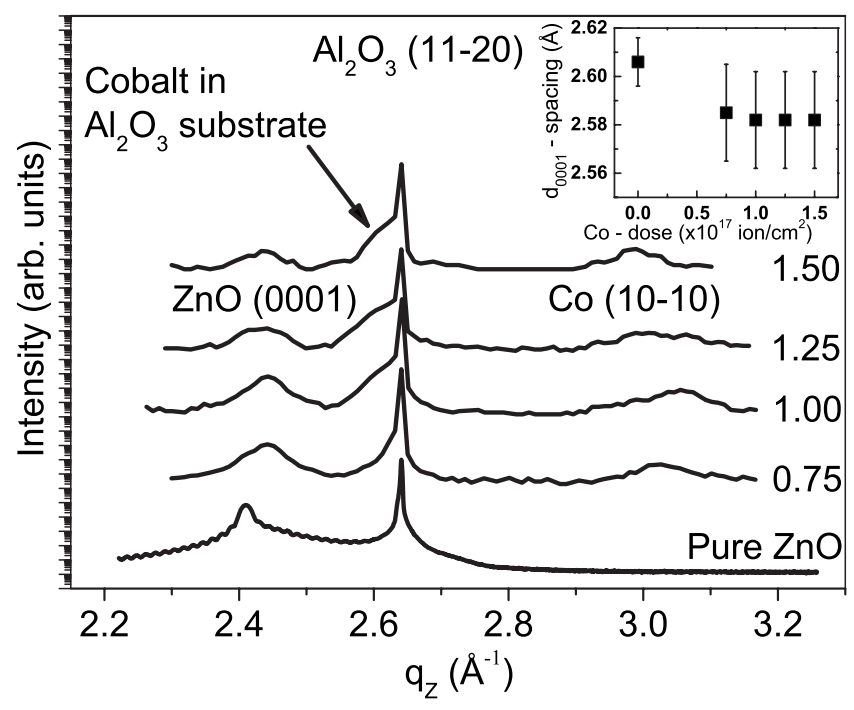

FIG. 3. High-angle Bragg scans of the $\mathrm{ZnO}(0001)$ films on $\mathrm{Al}_{2} \mathrm{O}_{3}(11 \overline{2} 0)$ before and after cobalt ion implantation. The inset shows the dependence of the $c$-axis spacing on the implantation dose.

used co-deposition during PLD for Co doping and observed a lattice expansion as a function of Co concentration. In both works the starting $c$-axis lattice parameters are 2.60 and $2.594 \AA$, which are contracted compared to the bulk value of $2.6035 \AA$ A. MacManus-Driscoll et al. $^{30}$ used a modified chemical-vapor deposition method for $\mathrm{ZnO}$ deposition and Co doping and they observed a $c$-axis expansion from $\mathrm{ZnO}$ $(2.604 \AA)$ to $\mathrm{Zn}_{0.98} \mathrm{Co}_{0.02} \mathrm{O}(2.605 \AA)$, followed by a contraction after an annealing step (2.603 and $2.604 \AA$, respectively). They explain the lattice contraction by removal of $\mathrm{Zn}$ interstitials upon annealing. In our case we start with a $c$-axis lattice parameter of $2.607 \AA$, which is then reduced to 2.585 $\AA$ upon Co doping. The increased lattice parameter of the virgin $\mathrm{ZnO}$ film may be due to epitaxial strain and $\mathrm{Zn}$ interstitials. An enhanced $c$-axis lattice parameter for $\mathrm{ZnO}(0001)$ on $\mathrm{Al}_{2} \mathrm{O}_{3}(11 \overline{2} 0)$ has been observed before (Ay et al. ${ }^{28}$ ). Cobalt doping by ion bombardment may remove strain and create oxygen vacancies. Both possibilities will reduce the lattice parameter. The analysis of the defect structure in the $\mathrm{ZnO}$ films is rather complex and will be investigated in more detail in the future. In addition, after implantation a tail (shown by an arrow in Fig. 3) appears on the low angle side of the main $\mathrm{Al}_{2} \mathrm{O}_{3}(11 \overline{2} 0)$ peak which is not observed before implantation. This tail likely reflects the lattice expansion of the sapphire substrate upon Co implantation.

In addition to the XRD experiments, we have also performed high-resolution cross sectional transmission electron microscopy (TEM) measurements for sample $6 .{ }^{19}$ The TEM results reveal the presence of metallic cobalt clusters in the $\mathrm{Al}_{2} \mathrm{O}_{3}$ sapphire substrate but not in the $\mathrm{ZnO}$ film. Co clusters with a diameter of about 5-6 $\mathrm{nm}$ form a Co rich layer in the substrate close to the $\mathrm{ZnO} / \mathrm{Al}_{2} \mathrm{O}_{3}$ interface. ${ }^{19}$

\section{B. Magnetic properties}

\section{Room temperature magnetization measurements}

For the investigation of the magnetic properties of the Co-implanted samples we used a high-resolution MOKE

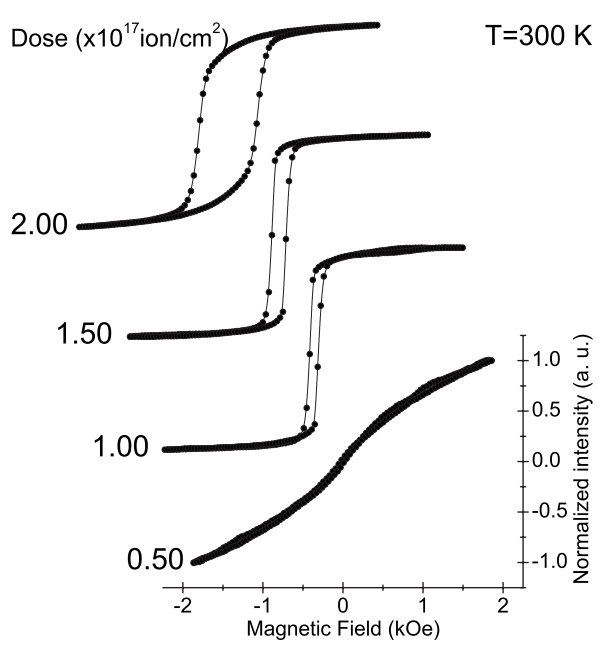

FIG. 4. Room temperature MOKE hysteresis curves of Co-implanted $\mathrm{ZnO}$ films measured for varying implantation dose.

setup in the longitudinal configuration with $s$-polarized light. $^{31-33}$ Figure 4 shows the hysteresis loops of Coimplanted $\mathrm{ZnO}$ films which were recorded at room temperature. The MOKE data in Fig. 4 clearly indicate that after cobalt implantation, nonmagnetic $\mathrm{ZnO}$ becomes ferromagnetic at room temperature with a large remanent magnetization. With increasing cobalt concentration the implanted $\mathrm{ZnO}$ films exhibit sequentially paramagnetic, weak ferromagnetic, and, finally, ferromagnetic responses with a square-like hysteresis at room temperature for the dose of 1.50 $\times 10^{17}$ ions $\mathrm{cm}^{-2}$. For the highest dose $(2.00$ $\times 10^{17}$ ions $\mathrm{cm}^{-2}$ ) the square-like shape of the hysteresis loop disappears and the coercive field increases drastically. From this we infer that for the highest dose level the cobalt atoms start to form clusters in the $\mathrm{ZnO}$ film. Moreover, although no in-plane magnetic anisotropy was observed by MOKE in Co-implanted $\mathrm{ZnO}$ films, we observed a clear sixfold in-plane magnetic anisotropy by ferromagnetic resonance (FMR) technique. ${ }^{34}$ The corresponding FMR data show that the easy and hard axes have a periodicity of $60^{\circ}$ in the film plane, in agreement with the hexagonal structure of the $\mathrm{ZnO}$ film.

In order to study in detail the observed ferromagnetic behavior, the magnetic properties of Co-implanted $\mathrm{ZnO}$ films were investigated using the XRMS technique. XRMS has proven to be a highly effective method for the analysis of the magnetic properties of buried layers and interfaces, including their depth dependence. ${ }^{35,36}$ Moreover, if the photon energy is fixed close to the energy of the corresponding x-ray absorption edges, element specific hysteresis loops can be measured. ${ }^{37}$ Since there are three elements in the Co-doped $\mathrm{ZnO}$ film, the analysis can be carried out separately for Co, $\mathrm{O}$, and $\mathrm{Zn}$. Furthermore, XRMS is surface sensitive but less than X-ray magnetic circular dichroism (XMCD). As photon in-photon out experiment, XRMS is very reliable independent of a possible surface contamination. Unless magnetic moments and separation of spin and orbital components are required, we prefer XRMS over XMCD. The penetration depth of soft $x$ rays depends on the energy difference to the resonance lines. We performed most of our XRMS experi- 


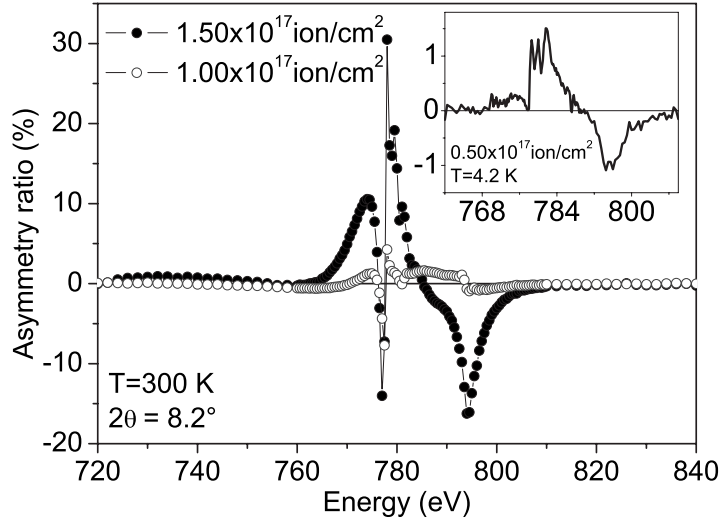

FIG. 5. The asymmetry ratios taken at the Co $L$ edges for sample $6(1.50$ $\times 10^{17}$ ions $\left.\mathrm{cm}^{-2}\right)$ and sample $4\left(1.00 \times 10^{17}\right.$ ions $\left.\mathrm{cm}^{-2}\right)$ shown by black and open symbols, respectively. Inset presents the asymmetry ratio of sample $2\left(0.50 \times 10^{17}\right.$ ions $\left.\mathrm{cm}^{-2}\right)$ measured at $4.2 \mathrm{~K}$.

ments close to $\mathrm{Co}_{3} L_{3}$ edge at $780 \mathrm{eV}$, for which the imaginary part of the refractive index has the value $1 \times 10^{-3}$. From this value we estimate a penetration depth of $100 \mathrm{~nm}$.

The XRMS experiments were performed using the ALICE diffractometer ${ }^{38}$ at the undulator beamline UE56/1-PGM at BESSY II (Berlin, Germany). The diffractometer comprises of a two-circle goniometer and works in horizontal scattering geometry. A magnetic field can be applied in the scattering plane and along the sample surface either parallel or antiparallel to the photon helicity, which corresponds to the longitudinal MOKE geometry. The maximum field of \pm 2700 Oe was high enough to fully saturate the magnetization of the sample. The magnetic contribution to the scattered intensity was always measured by reversing the magnetic field at fixed photon helicity. As a compromise between high scattering intensity and high magnetic sensitivity for the investigation of the magnetic properties at the Co $L$ edges, the scattering angle was fixed at the position of $2 \theta=8.2^{\circ}$ (the angle of incidence is $\theta=4.1^{\circ}$ ). ${ }^{19}$

The magnetic contribution to the resonant scattering can be best visualized by plotting the asymmetry ratio, $A_{r}=\left(I^{+}\right.$ $\left.-I^{-}\right) /\left(I^{+}+I^{-}\right)$. In Fig. 5 we show the asymmetry ratio taken at the Co $L$ edges for samples doped with different doses. The asymmetry ratio shows a strong ferromagnetic signal for sample 6 (up to $30 \%$ ), and it decreases with decreasing cobalt implantation dose. For sample 2, we observe only a very small magnetic signal at $4.2 \mathrm{~K}$. In addition to XRMS, we have also employed $x$-ray absorption spectroscopy (XAS) experiments for sample 6 . The XAS spectrum clearly exhibits a multiplet structure of the $\mathrm{Co}_{3}$ peak, which is typical for oxidized cobalt showing the presence of $\mathrm{Co}^{2+}$ state in the $\mathrm{ZnO}$ film. ${ }^{19}$

The magnetic signals at the $\mathrm{Zn} L_{3}(E=1021.8 \mathrm{eV})$ and the $\mathrm{O} K(526.8 \mathrm{eV})$ edges were also investigated. Within the sensitivity limit no magnetic signal could be resolved for $\mathrm{Zn}$. However, a clear magnetic signal was observed at the $\mathrm{O} K$ edge for sample $6 .{ }^{19}$ In addition to sample 6 , a very small magnetic signal at the $\mathrm{O} K$ edge was also observed for samples 4 and 7 presented in Fig. 6. The data shown in Fig. 6 have a low intensity asymmetry with a high level of noise for sample 4. However, important is the fact that the coer-

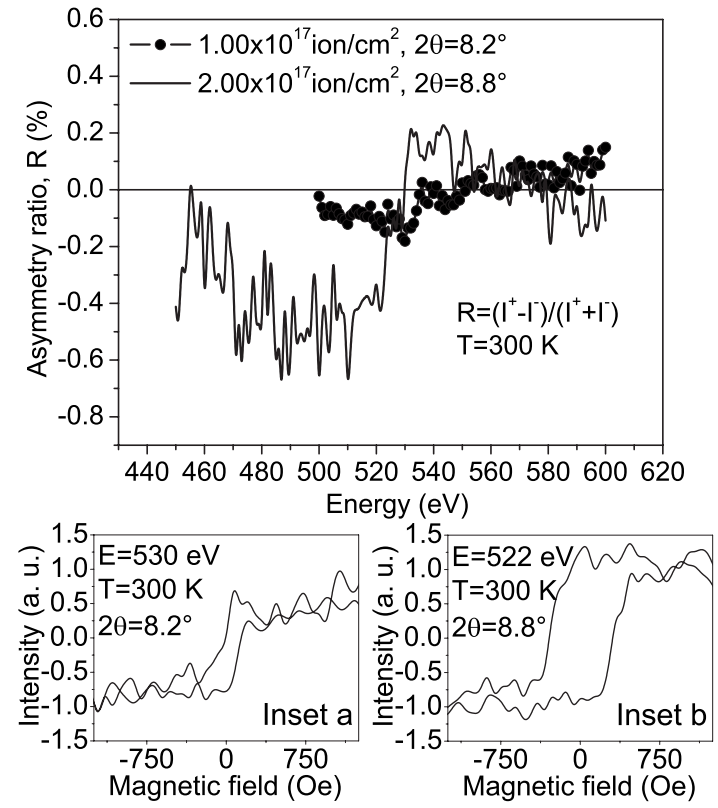

FIG. 6. The magnetic signal at the $\mathrm{O} K$ edge for samples 4 (black symbols) and 7 (solid line). Insets a and b show the hysteresis curves taken at the $\mathrm{O} K$ edge for samples 4 and 7 , respectively.

civities determined at the oxygen $K$ edge agree with those taken with a completely different and nonelement selective MOKE method. Thus the hysteresis measurements at the $\mathrm{O} K$ edge support the fact that oxygen in proximity to Co becomes polarized.

\section{Temperature dependent magnetization measurements}

In order to check the temperature dependence of the magnetization for $\mathrm{ZnO}$ films doped with different doses, we carried out field cooled (FC) and zero FC (ZFC) $M-T$ measurements using a SQUID magnetometer. For ZFC measurements, the samples are first cooled in zero field to $5 \mathrm{~K}$ and the magnetization is recorded during warming up to $390 \mathrm{~K}$ with an applied field of 100 Oe, parallel to the film surface. For FC measurements, the applied field of 100 Oe is kept constant during cooling to $5 \mathrm{~K}$ and the magnetization is recorded during warming at the same field value. Due to the clustering of cobalt in the $\mathrm{Al}_{2} \mathrm{O}_{3}$ substrate, ${ }^{19}$ the $\mathrm{FC}$ (closed symbols) and ZFC (open symbols) curves presented in Fig. 7 always show evidence for the presence of a superparamagnetic phase. There is a small peak at about $20 \mathrm{~K}$ in ZFC curve of sample $1\left(0.25 \times 10^{17}\right.$ ions $\left.\mathrm{cm}^{-2}\right)$ and this peak shifts to higher temperatures with increasing cobalt concentration. The trend in the $M-T$ curve of sample $1(0.25$ $\times 10^{17}$ ions $\mathrm{cm}^{-2}$ ) can be attributed to the coexistence of a ferromagnetic phase originating from substituted $\mathrm{Co}^{2+}$ ions in $\mathrm{ZnO}$ and the superparamagnetic phase due to cluster formation in $\mathrm{Al}_{2} \mathrm{O}_{3}$. Hysteresis curves measured at $5 \mathrm{~K}$ (Fig. 8) indicate that the superparamagnetic phase in this sample is more dominant than the ferromagnetic phase. The $M-T$ measurements for the samples implanted with higher doses exhibit superparamagnetism with a blocking temperature of about 100 and $250 \mathrm{~K}$ for sample $2\left(0.50 \times 10^{17}\right.$ ions $\left.\mathrm{cm}^{-2}\right)$ and sample $3\left(0.75 \times 10^{17}\right.$ ions $\left.\mathrm{cm}^{-2}\right)$, respectively. The hys- 


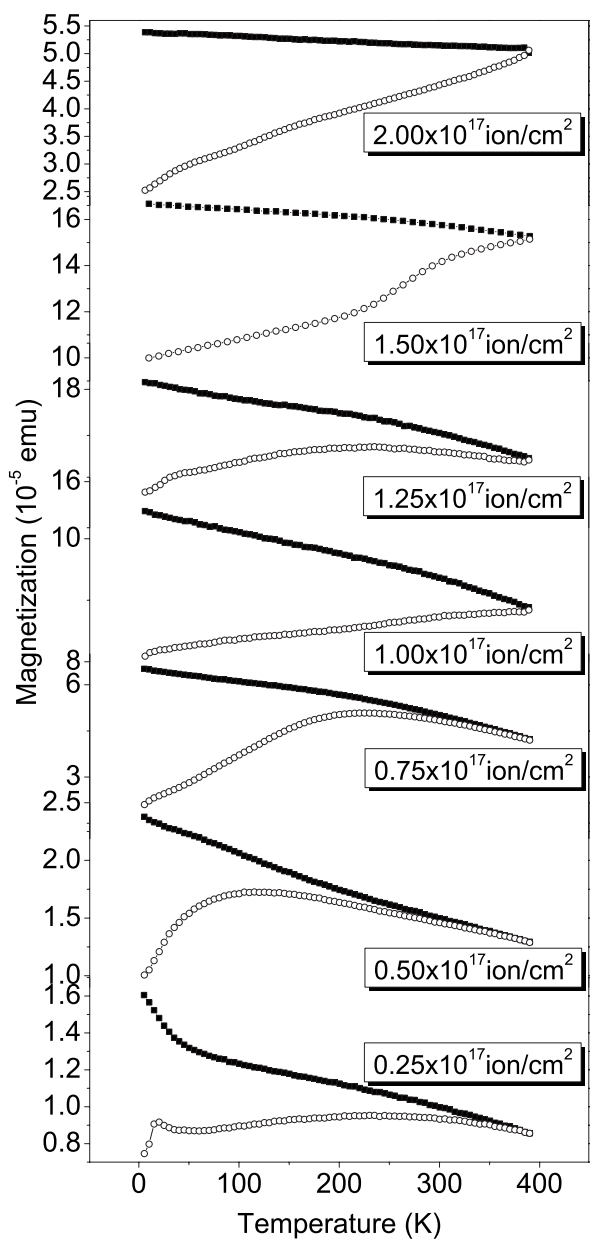

FIG. 7. Temperature dependent magnetization curves of Co-implanted $\mathrm{ZnO}$ films recorded by SQUID magnetometry for varying implantation dose. FC and ZFC curves refer to field cooled and zero-field cooled protocols and are presented by closed and open symbols, respectively. In both cases the data were taken in a field of 100 Oe during the heating up cycle.

teresis curves of these films (Fig. 8) also show that the superparamagnetic phase is still dominating over the ferromagnetic phase. But the steep part of the hysteresis curve of sample 3 and the increased coercivity $(0.75$ $\times 10^{17}$ ions $\mathrm{cm}^{-2}$ ) are indicative for the onset of a clear ferromagnetism phase at this dose. The temperature dependent magnetization curves of sample $4\left(1.00 \times 10^{17}\right.$ ions $\left.\mathrm{cm}^{-2}\right)$, sample $5\left(1.25 \times 10^{17}\right.$ ions $\left.\mathrm{cm}^{-2}\right)$, and sample $6 \quad 1.50$ $\times 10^{17}$ ions $\mathrm{cm}^{-2}$ show that these samples have a blocking

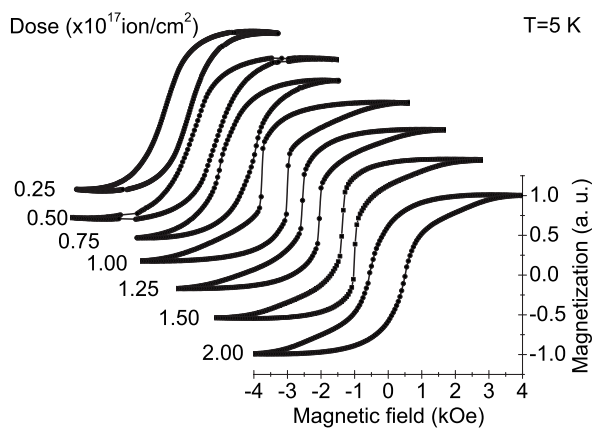

FIG. 8. SQUID $M-H$ loops of Co-implanted ZnO films measured for different implantation doses at $5 \mathrm{~K}$.

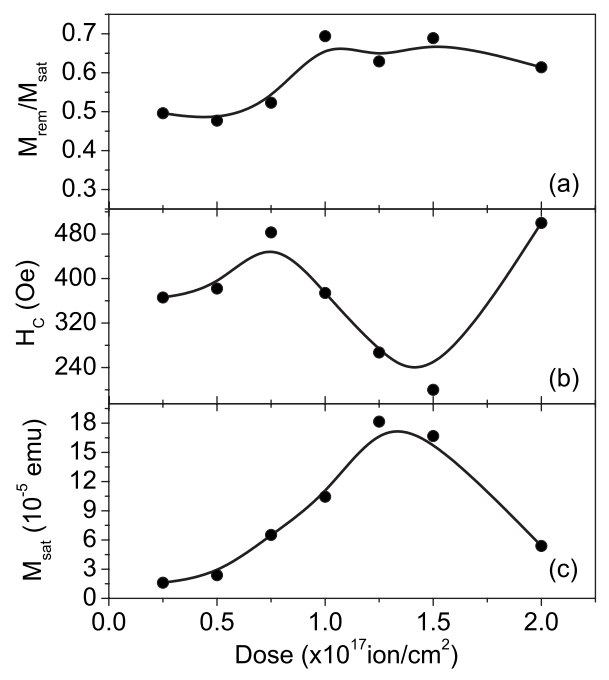

FIG. 9. The dose dependence of the normalized remanent magnetization (a), the coercive field (b), and the saturation magnetization (c). The data taken at $5 \mathrm{~K}$ using SQUID magnetometry.

temperature of about $390 \mathrm{~K}$ or even higher. The magnetic hysteresis of these samples measured by SQUID (Fig. 8) clearly shows a ferromagnetic phase superimposed by a superparamagnetic component. The ferromagnetic component is present even above room temperature as seen in the MOKE experiments in Fig. 4. Since MOKE probes only films near their surface, the superparamagnetic component in these samples, which is deeper in the substrate, is not seen by MOKE experiments.

In the SQUID hysteresis curves there is another remarkable effect of the ferromagnetic phase as a function of dose. The coercivity $H_{C}$ decreases systematically with increasing Co dose up until a dose of $1.50 \times 10^{17}$ ions $\mathrm{cm}^{-2}$, as seen in Figs. 8 and 9(b). This behavior may be explained as follows: with increasing Co dose the magnetization becomes more homogeneous and, since magnetic inhomogeneities are the main source of pinning for the domain walls, $H_{C}$ decreases with increasing Co dose. Between $1.25 \times 10^{17}$ and 1.50 $\times 10^{17}$ ions $\mathrm{cm}^{-2}$ the potential barrier for reversal of the ferromagnetic component becomes smaller. Up to this level all inhomogeneities are filled. Any higher dose is counterproductive; it decreases the saturation magnetization and enhances the coercivity [see Figs. 9(b) and 9(c)], indicating that Co goes into antisites with eventually antiferromagnetic (AF) coupling, loss of magnetization, and increase in the coercivity. $\mathrm{CoO}$ clusters are formed in the $\mathrm{ZnO}$ matrix with $\mathrm{AF}$ spin structure and $\mathrm{AF}$ coupling to the remaining ferromagnetic $\mathrm{Zn}(\mathrm{Co}) \mathrm{O}$ film. The $M-T$ data (Fig. 7) and the room temperature (Fig. 4) and low temperature (Fig. 8) hysteresis measurements of sample $7\left(2.00 \times 10^{17}\right.$ ions $\left.\mathrm{cm}^{-2}\right)$ clearly indicate that the cobalt atoms start to cluster also within the $\mathrm{ZnO}$ layer at the highest dose.

\section{Hall effect measurements}

In ferromagnetic materials the Hall voltage consists of the ordinary term and an additional term that contributes to the Hall voltage due to their spontaneous magnetization. This 


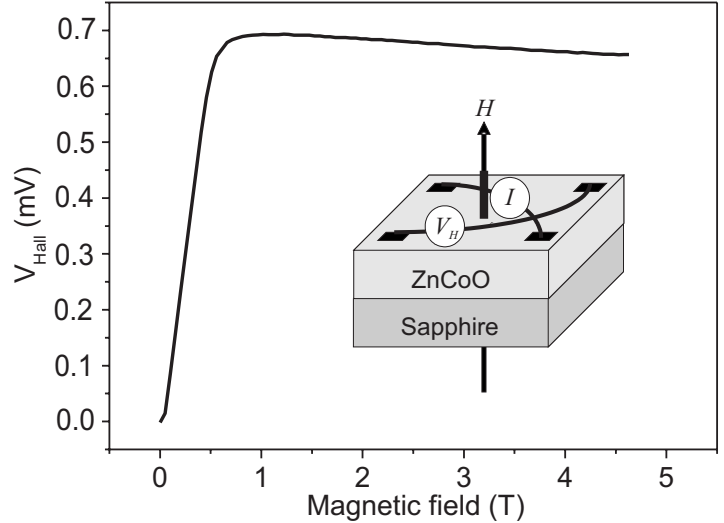

FIG. 10. AHE data of sample $6\left(1.50 \times 10^{17}\right.$ ions $\left.\mathrm{cm}^{-2}\right)$ taken at $4.2 \mathrm{~K}$. Inset shows the geometry of the AHE measurements. $H$ is the external magnetic field applied perpendicular to the film surface.

additional contribution, called anomalous Hall effect (AHE), is proportional to the sample magnetization. ${ }^{39}$ Hence, the Hall voltage can be written as ${ }^{39}$

$$
V_{H}=\left(\frac{R_{0} I}{t}\right) H \cos \alpha+\left(\frac{R_{A} \mu_{0} I}{t}\right) M \cos \theta,
$$

where $t$ is the film thickness and $I$ is the current. $R_{0}$ and $R_{A}$ are the ordinary and anomalous Hall effect coefficients, respectively. $\mu_{0}$ is the permeability of free space, $\alpha$ is the angle between the applied magnetic field $(H)$ and sample normal, and $\theta$ is the angle between the sample magnetization $(M)$ and the sample normal. The first term in Eq. (1) is the ordinary Hall effect and arises from the Lorentz force acting on conduction electrons. This establishes an electric field perpendicular to the applied magnetic field and to the current. The anomalous Hall effect term is conventionally attributed to spin dependent scattering involving a spin-orbit interaction between the conduction electrons and the magnetic moments of the material. At low applied magnetic fields, the Hall voltage $\left(V_{H}\right)$ is dominated by the magnetic field dependence of the sample magnetization $M$. When the applied magnetic field is high enough to saturate the sample magnetization, the magnetic field dependence of the Hall voltage becomes linear due to the ordinary Hall effect.

In order to check whether this behavior is present in Co-implanted $\mathrm{ZnO}$ films and to determine the character of the majority carriers, we have carried out Hall effect experiments. The Hall effect measurements were performed at 4.2 $\mathrm{K}$ using a van der Pauw configuration presented in Fig. 10 as an inset.

The Hall effect data of sample $6 \quad(1.50$ $\times 10^{17}$ ions $\mathrm{cm}^{-2}$ ) are shown in Fig. 10. A sharp rise in the Hall voltage at low field, i.e., AHE, is followed by a slow decrease corresponding to the ordinary Hall effect. It is important to note that the negative slope at high fields indicates $n$-type carriers in Co-implanted $\mathrm{ZnO}$ film with a three dimensional carrier concentration of $n_{3 \mathrm{D}}=1.931 \times 10^{19} \mathrm{~cm}^{-3}$. The Hall mobility measured at $4.2 \mathrm{~K}$ is about $90 \mathrm{~cm}^{2} \mathrm{~V}^{-1} \mathrm{~s}^{-1}$ for sample 6. We have also observed similar behavior for the samples 3-5 and 7. However, for the lowest two doses (samples 1 and 2), the measurements cannot be done because of a too small signal-to-noise ratio of the Hall voltage.

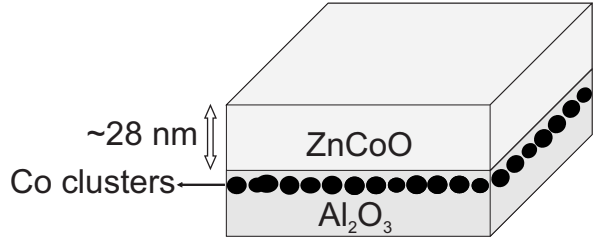

FIG. 11. The cluster formation in $\mathrm{Al}_{2} \mathrm{O}_{3}$ substrate after cobalt ion implantation.

\section{DISCUSSION}

For the dose dependence of magnetic phases in $\mathrm{ZnO}$ films at room temperature we propose the following scenario: at low doses $\left[(0.25-0.50) \times 10^{17}\right.$ ions $\left.\mathrm{cm}^{-2}\right]$ the number of substituted cobalt ions in the $\mathrm{ZnO}$ layer is very small, which results in a paramagnetic signal at room temperature. Increasing of cobalt implantation dose leads to an increasing number of substituted cobalt ions and after certain cobalt concentration they start to interact ferromagnetically. For this reason at the cobalt dose of $0.75 \times 10^{17}$ ions $\mathrm{cm}^{-2}$ a weak ferromagnetic behavior is observed with a $T_{c}$ below room temperature. At higher cobalt concentrations [(1.00-1.50) $\times 10^{17}$ ions $\left.\mathrm{cm}^{-2}\right]$ the substituted cobalt ions in $\mathrm{ZnO}$ interact strongly and stabilize room temperature ferromagnetism. At the highest dose of $2.00 \times 10^{17}$ ions $\mathrm{cm}^{-2}$, in addition to the substituted cobalt ions, metallic cobalt clusters are also present in the $\mathrm{ZnO}$ layer.

As discussed in detail in Ref. 19, the difference in the shape of the hysteresis loops obtained by MOKE and SQUID is attributed to the surface sensitivity of the MOKE technique. MOKE is particularly useful in the study of surface magnetism since it is highly sensitive to the magnetization within the skin depth region, typically $10-20 \mathrm{~nm}$ in most metals. ${ }^{40} \mathrm{ZnO}$ is transparent to visible light before cobalt implantation. However, after doping with cobalt atoms at high concentrations (50\%), it becomes metallic-like and reflects the laser beam $(\lambda=632 \mathrm{~nm})$ from the surface of the $\mathrm{Co} \mathrm{ZnO}$ films. Thus at high concentrations, $\mathrm{ZnCoO}$ behaves like a TM with a short penetration depth. The $\mathrm{ZnO}$ films have a thickness of $35 \mathrm{~nm}$ before implantation. Because of the surface sputtering, the $\mathrm{ZnO}$ thickness decreases (e.g., decreased to about $28 \mathrm{~nm}$ for sample 6) after implantation. Thus, MOKE provides information only from the $\mathrm{ZnO}$ layer not from the sapphire substrate, i.e., MOKE is only sensitive to the ferromagnetic contribution from the $\mathrm{ZnO}$ layer. In this layer some portion of nonmagnetic $\mathrm{Zn}$ atoms is substituted by magnetic Co ions, giving raise to the MOKE hysteresis. However, SQUID measurements collect magnetic contributions from both the Co-implanted $\mathrm{ZnO}$ film and from the cobalt clusters in $\mathrm{Al}_{2} \mathrm{O}_{3}$ (Fig. 11). Therefore, the difference between the MOKE and SQUID data appears as a result of the depth-dependent Co content in the implanted layer.

Another important result of this study is the observation of oxygen spin polarization in Co-implanted $\mathrm{ZnO}$ films. Oxygen is present both in $\mathrm{ZnO}$ and in the sapphire substrate. At the oxygen $K$ edge, the x-ray penetration is however very small on the order of the film thickness, such that the substrate cannot be seen. This shows that the oxygen atoms are polarized due to the spontaneous ferromagnetic order in $\mathrm{ZnO}$ 
films. The main question that arises here is the mechanism which leads to the observed long range ferromagnetic ordering in Co-doped $\mathrm{ZnO}$. Recently, Patterson ${ }^{41}$ calculated the electronic band structure of Co substituted for $\mathrm{Zn}$ in $\mathrm{ZnO}$, for $\mathrm{Zn}$ and $\mathrm{O}$ vacancies, and for interstitial $\mathrm{Zn}$ in $\mathrm{ZnO}$ using the B3LYP hybrid density functional theory. He reported that the singly-positively charged $\mathrm{O}$ vacancy is the only defect in Co-doped $\mathrm{ZnO}$ which can mediate ferromagnetic exchange coupling between Co ions at intermediate range (just beyond near neighbor distances). In the ground state configuration the majority Co spins are parallel, whereas the minority spins are parallel to each other and to the oxygen vacancy spin, so that there are exchange couplings between these three spins leading to an overall ferromagnetic ground state of the Co ions. No substantial exchange coupling was found for the positively charged interstitial $\mathrm{Zn}$ defect which has also spin $1 / 2$. The exchange coupling mechanism proposed by Patterson is essentially the same as the impurity band model of Coey et al. ${ }^{42}$ where the polarons bound to the oxygen vacancies mediate ferromagnetic coupling between Co ions. In order to have the magnetic moments of the Co ions aligned ferromagnetically, one mediating electron is required with an oppositely directed spin. This is in line with a recent comparison of band structure calculations by Walsh $e t a l .{ }^{43}$ showing that the electronic structure of Co-doped $\mathrm{ZnO}$ is consistent with carrier mediated ferromagnetism. The oxygen spin polarization has not explicitly been considered in the aforementioned band structure calculations and may be due to ferromagnetic splitting of nearest neighbor oxygen $p$-levels. This has already been speculated by Methfessel and Mattis ${ }^{44}$ in their seminal review article on magnetic semiconductors.

The reason for the observation of AHE and $n$-type carriers in Co-implanted $\mathrm{ZnO}$ films can be explained by electron doping via $\mathrm{Zn}$ interstitials. Normally, isovalent $\mathrm{TM}^{2+}$ doping of $\mathrm{ZnO}$ does not introduce charge carriers itself; they need to be produced by additional doping. ${ }^{45}$ However, using ion implantation not only cobalt ions are introduced in $\mathrm{ZnO}$ but simultaneously many other defects are also be produced in the implanted region, such as $\mathrm{Zn}$ interstitials which are reported to form shallow donors in $\mathrm{ZnO} .^{17,46,47}$ This can be thought of as an added advantage of ion implantation that it not only introduces TM ions to induce ferromagnetism but also introduces the required charge carriers into the $\mathrm{ZnO}$.

\section{SUMMARY}

In conclusion, the structural, magnetic, and electronic properties of Co-implanted $\mathrm{ZnO}$ films, deposited by rfsputtering methods on a $(11 \overline{2} 0)$ oriented sapphire substrate, have been investigated. The structural data indicate a Co cluster formation in the sapphire substrate close to the $\mathrm{ZnO} / \mathrm{Al}_{2} \mathrm{O}_{3}$ interface but well separated from the $\mathrm{ZnO}$ film. No indication of clustering in the $\mathrm{ZnO}$ layer has been found. The previously reported XAS data with a multiplet fine structure around the Co $L_{3}$ edge clearly show that the implanted cobalt ions are in the $\mathrm{Co}^{2+}$ oxidation state, most likely substituting part of the $\mathrm{Zn}^{2+}$ ions in the host matrix. The combination of room temperature and low temperature magnetization measurements indicates that there are two magnetic phases in the Co-implanted $\mathrm{ZnO} / \mathrm{Al}_{2} \mathrm{O}_{3}$ films. One is the ferromagnetic phase due to the Co substitution on $\mathrm{Zn}$ sites in the $\mathrm{ZnO}$ film and the second magnetic phase originates from Co clusters in the sapphire substrate. Furthermore, a clear ferromagnetic signal at the $\mathrm{O} K$ edge is observed which shows that the oxygen spin polarization is an important indicator for the observed long range ferromagnetic ordering in the $\mathrm{ZnO}$ layer. In conclusion, implantation of cobalt ions into the nonmagnetic $\mathrm{ZnO}$ film causes intrinsic ferromagnetism at room temperature and simultaneously creates $n$-type charge carriers without additional doping.

\section{ACKNOWLEDGMENTS}

We would like to acknowledge S. Erdt-Böhm and P. Stauche for sample preparation and technical support. We also would like to thank also Dr. C. Sternemann and Dr. M. Paulus for their assistance with the beamline operation at DELTA, and G. Nowak for his help to perform XRD experiments at HASYLAB. This work was partially supported by BMBF through Contract Nos. 05KS4PCA (ALICE Chamber) and 05ES3XBA/5 (Travel to BESSY), by DFG through Grant No. SFB 491, and by RFBR through Grant No. 07-0200559-a. N. Akdogan acknowledges a fellowship through the International Max Planck Research School-SurMat.

${ }^{1}$ R. W. G. Wyckoff, Crystal Structures, 2nd ed. (Wiley, New York, 2001). ${ }^{2}$ T. Dietl, H. Ohno, F. Matsukura, J. Cibert, and D. Ferrand, Science 287, 1019 (2000)

${ }^{3}$ K. Sato and H. Katayama-Yoshida, Jpn. J. Appl. Phys., Part 2 39, L555 (2000).

${ }^{4}$ K. Sato and H. Katayama-Yoshida, Jpn. J. Appl. Phys., Part 2 40, L334 (2001).

${ }^{5}$ K. Ueda, H. Tabada, and T. Kawai, Appl. Phys. Lett. 79, 988 (2001).

${ }^{6}$ S. W. Lim, S. K. Hwang, and J. M. Myoung, Solid State Commun. 125, 231 (2003).

${ }^{7}$ Y. Z. Yoo, T. Fukumura, Z. Jin, K. Hasagewa, M. Kawasaki, P. Ahmet, T. Chikyov, and H. Koinuma, J. Appl. Phys. 90, 4246 (2001).

${ }^{8}$ J. H. Kim, H. Kim, D. Kim, Y. E. Ihm, and W. K. Choo, J. Appl. Phys. 92, 6066 (2002).

${ }^{9}$ J. H. Kim, H. Kim, D. Kim, Y. E. Ihm, and W. K. Choo, Physica B 327, 304 (2003).

${ }^{10}$ W. Prellier, A. Fouchet, B. Mercey, C. Simon, and B. Raveau, Appl. Phys. Lett. 82, 3490 (2003).

${ }^{11}$ M. Venkatesan, C. B. Fitzgerald, J. G. Lunney, and J. M. D. Coey, Phys. Rev. Lett. 93, 177206 (2004).

${ }^{12}$ L. Yan, C. K. Ong, and X. S. Rao, J. Appl. Phys. 96, 508 (2004).

${ }^{13}$ S. Ramachandran, A. Tiwari, and J. Narayan, Appl. Phys. Lett. 84, 5255 (2004).

${ }^{14}$ M. Ivill, S. J. Pearton, S. Rawal, L. Leu, P. Sadik, R. Das, A. F. Hebard, M. Chisholm, J. D. Budai, and D. P. Norton, New J. Phys. 10, 065002 (2008).

${ }^{15}$ Z. Jin, T. Fukumura, M. Kawasaki, K. Ando, H. Saito, and T. Sekiguchi, Appl. Phys. Lett. 78, 3824 (2001).

${ }^{16}$ Y. Zheng, J. C. Boilliard, D. Demaille, Y. Bernard, and J. F. Petroff, J. Cryst. Growth 274, 156 (2005).

${ }^{17}$ H. J. Lee, S. Y. Jeong, C. R. Cho, and C. H. Park, Appl. Phys. Lett. 81, 4020 (2002).

${ }^{18}$ Y. He, P. Sharma, K. Biswas, E. Z. Liu, N. Ohtsu, A. Inoue, Y. Inada, M. Nomura, J. S. Tse, S. Yin, and J. Z. Jiang, Phys. Rev. B 78, 155202 (2008).

${ }^{19}$ N. Akdogan, A. Nefedov, K. Westerholt, H. Zabel, H. W. Becker, C. Somsen, R. Khaibullin, and L. Tagirov, J. Phys. D 41, 165001 (2008).

${ }^{20}$ T. Fukumura, J. Zin, A. Ohtomo, and H. Koinuma, Appl. Phys. Lett. 75, 3366 (1999).

${ }^{21}$ X. M. Cheng and C. L. Chien, J. Appl. Phys. 93, 7876 (2003).

${ }^{22}$ M. Chakrabarti, S. Dechoudhury, D. Sanyal, T. Roy, D. Bhowmick, and A. Charabarti, J. Phys. D 41, 135006 (2008).

${ }^{23}$ H. Saeki, H. Matsui, T. Kawai, and H. Tabata, J. Phys. Chem. 16, S5533 
(2004).

${ }^{24}$ Y. M. Cho, W. K. Choo, H. Kim, D. Kim, and Y. Ihm, Appl. Phys. Lett 80, 3358 (2002).

${ }^{25}$ J. H. Park, M. G. Kim, H. M. Jang, S. Ryu, and Y. M. Kim, Appl. Phys. Lett. 84, 1338 (2004).

${ }^{26}$ A. F. Hebard, R. P. Rairigh, J. G. Kelly, S. J. Pearton, C. R. Abernathy, S. N. G. Chu, and R. G. Wilson, J. Phys. D 37, 511 (2004).

${ }^{27}$ Z. Yin, N. Chen, C. Chai, and F. Yang, J. Appl. Phys. 96, 5093 (2004)

${ }^{28}$ M. Ay, A. Nefedov, and H. Zabel, Appl. Surf. Sci. 205, 329 (2003).

${ }^{29}$ J. F. Ziegler, J. P. Biersack, and U. Littmark, The Stopping and Range of Ions in Solids (Pergamon, New York, 1985).

${ }^{30}$ J. L. MacManus-Driscoll, N. Khare, Y. Liu, and M. E. Vickers, Adv. Mater. (Weinheim, Ger.) 19, 2925 (2007).

${ }^{31}$ T. Zeidler, F. Schreiber, H. Zabel, W. Donner, and N. Metoki, Phys. Rev. B 53, 3256 (1996)

${ }^{32}$ T. Schmitte, K. Westerholt, and H. Zabel, J. Appl. Phys. 92, 4524 (2002).

${ }^{33}$ A. Westphalen, M. S. Lee, A. Remhof, and H. Zabel, Rev. Sci. Instrum. 78, 121301 (2007).

${ }^{34}$ N. Akdogan, B. Rameev, S. Güler, A. Nefedov, H. Zabel, R. Khaibullin, and L. Tagirov (unpublished).

${ }^{35}$ J. M. Tonnerre, L. Seve, D. Raoux, G. Soullie, B. Rodmacq, and P. Wolfers, Phys. Rev. Lett. 75, 740 (1995).
${ }^{36}$ G. van der Laan, Curr. Opin. Solid State Mater. Sci. 10, 120 (2006).

${ }^{37}$ J. B. Kortright, J. S. Jiang, S. D. Bader, O. Hellwig, D. T. Marguiles, and E. E. Fullerton, Nucl. Instrum. Methods Phys. Res. B 199, 301 (2003).

${ }^{38}$ J. Grabis, A. Nefedov, and H. Zabel, Rev. Sci. Instrum. 74, 4048 (2003).

${ }^{39}$ C. M. Hurd, The Hall Effect in Metals and Alloys (Plenum, New York, 1972).

${ }^{40}$ J. A. C. Bland, M. J. Padgett, R. J. Butcher, and N. Bett, J. Phys. E 22, 308 (1989).

${ }^{41}$ C. H. Patterson, Phys. Rev. B 74, 144432 (2006).

${ }^{42}$ J. M. D. Coey, M. Venkatesan, and C. B. Fitzgerald, Nature Mater. 4, 173 (2005).

${ }^{43}$ A. Walsh, J. L. F. D. Silva, and S.-H. Wei, Phys. Rev. Lett. 100, 256401 (2008).

${ }^{44}$ S. Methfessel and D. Mattis, Encyclopedia of Physics, edited by S. Flügge, (Springer, New York, 1968), Vol. XVIII, p. 1.

${ }^{45}$ K. Nielsen, S. Bauer, M. Lübbe, S. T. B. Goennenwein, M. Opel, J. Simon, W. Mader, and R. Gross, Phys. Status Solidi A 203, 3581 (2006).

${ }^{46}$ D. C. Look, D. C. Reynolds, J. R. Sizelove, R. L. Jones, C. W. Litton, G. Cantwell, and W. C. Harsch, Solid State Commun. 105, 399 (1998).

${ }^{47}$ D. C. Look, J. W. Hemsky, and J. R. Sizelove, Phys. Rev. Lett. 82, 2552 (1999). 